\title{
A SIMPLIFIED MODEL OF QUADRATIC COST FUNCTION FOR THERMAL GENERATORS
}

\author{
ZIVIC DJUROVIC, M[arijana]; MILACIC, A[leksandar] \& KRSULJA, M[arko]
}

\begin{abstract}
The paper describes practical method for analyzing the economic dispatch of a power system using Lagrange multiplier method regarding Kuhn-Tucker conditions, without taking into consideration the transmission limitations and losses. The example describes nine thermal generators with different fuel cost functions. Generator curves are represented with quadratic fuel cost functions and with simplified, linear model. Optimal solution of power output from each generator is presented, regarding both cost functions in correlation to different values of load demand. The possibility of using described simplified cost functions in active distribution network is also suggested.
\end{abstract}

Keywords: lagrange multiplier, economic dispatch, cost function, optimization

\section{INTRODUCTION}

The decentralization of the electric power system with steady growth of demand for power and public pressure to reduce greenhouse gas emissions are reasons for increased use of distributed generation (DG). DG has a significant impact on the overall system operation and control especially when different DG units and storage devices are combined. In order to obtain an economically optimal operation of these units, a mathematical model and optimization is needed [1].

Optimization is finding the precise solution, of many existing solutions, that fulfills the maximum conditions, from one specific and established point of view. If that particular goal is oriented towards the economic domain, economic optimum represents that situation or state of economy that assures the highest efficiency [2]. Economic dispatch, as part of unit commitment, represents the scheduling of generators to minimize the total operating cost. Mathematically, it can be stated as a constrained optimization problem. In solutions to constrained problems the goal is to find a $m$ vector of choice variables $\mathbf{P}$ which minimizes $f(\mathbf{P})$ subject to:

$$
\begin{aligned}
& g_{i}(\mathbf{P}) \leq 0, \quad i=1, \ldots, n \\
& h_{j}(\mathbf{P})=0, \quad i=1, \ldots, r .
\end{aligned}
$$

In above equations, we have $n$ inequality constraints and $r$ equality constraints. Nonlinear constrained optimization is referred to as nonlinear programming [3].

There are many ways for defining the operating state of generators. The main goal is to include as many variables as possible that effect operational costs, such as the generator distance from the load, type of fuel, load capacity and transmission line losses.

The generator cost is typically represented by four curves: fuel cost, heat rate, input/output (I/O) and incremental cost. Generator curves are generally represented as cubic or quadratic functions and piecewise linear functions. Thermal power plant uses a quadratic fuel cost function such as the Fuel Cost Curve [4]:

$$
C_{i}\left(P_{G i}\right)=a+b P_{G i}+c P_{G i}^{2},
$$

where $i=$ unit number (generator); $C_{i}=$ operating cost of unit; $P_{G i}=$ electrical power output (power generation) of a unit $i ; a, b$ and $c=$ fuel cost coefficients of unit $i$. The fuel cost curve allows us to look at a wide range of economic dispatch practice such as total operating cost of a system, incremental cost and minute by minute loading of a generator. The fuel cost function becomes more nonlinear when the actual generator response is considered. Quadratic and naturally, cubic cost functions more accurately models the actual response of conventional thermal generators where fuel is oil, coal and gas, but also diesel generators, gas micro turbines, biomass power plants, fuel cells, etc [5]. Energy sources such as solar, wind and hydro are not included because the fuel that drives its power generation is without price.

The main elements that are used to define the cost of electrical energy generation are: operating costs, facility construction and ownership cost [4]. The operating cost is the most significant one and it is dominated mostly by the fuel cost. The aim of power system economic dispatch is to maximize system efficiency and minimize system losses that can't be billed or pass on to customers. Input/output test and calculations are used to provide system performance data in the form of input and output equations. I/O curves are primarily used in the calculation of the total incremental cost. Incremental cost is the inclination of the fuel cost curve and it represents the cost of next unit of energy from that generator at a specific output level $P_{G i}$ of the $i^{\text {th }}$ generator. In short, it describes how much it will cost to operate a generator to produce an additional unit of power.

$$
I C_{i}=\lambda=\frac{\partial C_{i}\left(P_{G i}\right)}{\partial P_{G i}},
$$

Most economic dispatch algorithms when subjected to equality and inequality constraints use Lagrange multipliers to set up the optimization problem [6]. Several standard search techniques like lambda iteration method [4], gradient search method, the Newton Raphson iteration method, secant method [7] and linear programming have been developed to solve comparable optimization problems. Nevertheless, these methods have certain limitations on solving constrained optimizations. 


\section{PROBLEM FORMULATION}

Problem formulation is to find the optimal output $P_{i}$ to minimize the cost of power generation from nine generators with different cost functions to supply specific load demand $P_{G D}$. The objective function to minimize is:

$$
\mathrm{f}\left(\mathrm{P}_{\mathrm{Gi}}\right)=\sum_{\mathrm{i}=1}^{9} C_{i}\left(P_{G i}\right)
$$

Cost function coefficients of nine generators according to (3) and generator output limits (constraints) are presented in table 1. The original data are from [7], [8] and [9]. The units are arranged by size, according to their minimum and maximum constraints, the smallest one first.

The condition that the total load be equal to specific power demand $P_{G D}$ is an equality constraint because:

$$
\sum_{i=1}^{9} P_{G i}=P_{G D}
$$

The power output of any generator must not exceed its rating nor drop below a given value for stable operation. In minimizing cost of generating electrical power without compromising system reliability and market security, inequality constraints of each generator must also be defined:

$$
P_{i}^{\min } \leq P_{G i} \leq P_{i}^{\max },
$$

The upper boundary $P_{i}^{\max }$ is directly related to upper rating of the generator while lower boundary $P_{i}^{\min }$ is directly linked to thermal consideration that is required to maintain the steam which drives the turbine (table 1).

In the following theoretical development, we convert all constraints to equalities. In order to do so, we add an appropriate non-negative slack variables $y_{i}{ }^{2}$ and $x_{i}{ }^{2}$, the values of which are yet unknown [3]. Converting all defined inequalities to equalities, one gets:

$$
\begin{aligned}
& P_{i}^{\min }-P_{G i}+y_{i}{ }^{2}=0, \quad i=1, \ldots, 9, \\
& P_{G i}-P_{i}^{\max }+x_{i}{ }^{2}=0, \quad i=1, \ldots, 9,
\end{aligned}
$$

where $y_{i}$ and $x_{i}$ are artificially introduced variables.

In order to minimize $f(\mathbf{P})$, we minimize the Lagrange function defined as:

$$
L(\mathbf{P}, \mathbf{x}, \mathbf{y}, \boldsymbol{\lambda}, \boldsymbol{\beta})=f(\mathbf{P})+\sum_{i=1}^{n} \lambda_{i} g_{i}(\mathbf{P}, \mathbf{x}, \mathbf{y})+\sum_{k=1}^{r} \beta_{k} h_{k}(\mathbf{P}, \mathbf{x}, \mathbf{y}),
$$

where $\lambda$ is a vector of Lagrange multipliers for inequalities, and $\boldsymbol{\beta}$ is a vector of Lagrange multipliers for equalities. In order to minimize our objected function (5), we minimize the Lagrangian function obtained as:

$$
\begin{aligned}
& L\left(P_{G i}, y_{i}, x_{i}, \lambda, \beta_{i}{ }^{\min }, \beta_{i}{ }^{\max }\right)=f\left(P_{G i}\right)+\lambda \cdot\left(P_{G D}-\sum_{i=1}^{9} P_{G i}\right)+ \\
& +\sum_{i=1}^{9} \beta_{i}{ }^{\min }\left(P_{i}{ }^{\min }-P_{G i}+y_{i}{ }^{2}\right)+\sum_{i=1}^{9} \beta_{i}{ }^{\max }\left(P_{G i}-P_{i}^{\max }+x_{i}{ }^{2}\right),
\end{aligned}
$$

where $\lambda, \beta_{i}^{\text {min }}$ and $\beta_{i}^{\max }$ are the Lagrange multipliers associated with the constraint of total demand, minimum and maximum output from the generators.

\begin{tabular}{|c|c|c|c|c|c|}
\hline \multirow{2}{*}{$\begin{array}{c}\text { Unit } \\
\boldsymbol{i}\end{array}$} & \multicolumn{2}{|c|}{ Cost function coefficients } & \multicolumn{2}{c|}{ Constraints } \\
\cline { 2 - 6 } & $\boldsymbol{a}_{\boldsymbol{i}}$ & $\boldsymbol{b}_{\boldsymbol{i}}$ & $\boldsymbol{c}_{\boldsymbol{i}}$ & $\boldsymbol{P}_{\boldsymbol{i}}^{\text {min }}[\mathrm{MW}]$ & $\boldsymbol{P}_{\boldsymbol{i}}^{\boldsymbol{m a x}^{2}}[\mathrm{MW}]$ \\
\hline 1 & 24.3891 & 25.5472 & 0.02533 & 2.4 & 12 \\
\hline 2 & 117.7551 & 37.5510 & 0.01199 & 4 & 20 \\
\hline 3 & 100 & 6 & 0.005 & 7 & 28 \\
\hline 4 & 660 & 25.92 & 0.00413 & 10 & 55 \\
\hline 5 & 300 & 8 & 0.0025 & 14 & 56 \\
\hline 6 & 81.1364 & 13.3272 & 0.00876 & 15.2 & 76 \\
\hline 7 & 500 & 10 & 0.002 & 20 & 84 \\
\hline 8 & 217.8952 & 18 & 0.00623 & 25 & 100 \\
\hline 9 & 680 & 16.5 & 0.00211 & 20 & 130 \\
\hline
\end{tabular}

Tab. 1. Cost functions coefficients and constraints of 9 generators

The Kuhn-Tucker necessary condition requires that the gradient of $L$ be zero [3]. Hence, we want:

$$
\nabla L=\left[\begin{array}{l}
\partial L / \partial P_{G i} \\
\partial L / \partial y_{i} \\
\partial L / \partial x_{i} \\
\partial L / \partial \lambda \\
\partial L / \partial \beta_{i}{ }^{\min } \\
\partial L / \partial \beta_{i}{ }^{\max }
\end{array}\right]=0, \quad i=1, \ldots, 9,
$$

In order to satisfy the (11), the vector to be solved for is:

$$
z=\left[P_{G i}, y_{i}, x_{i}, \lambda, \beta_{i}{ }^{\min }, \beta_{i}{ }^{\max }\right]^{T}, \quad i=1, \ldots, 9 .
$$

\section{DISCUSSION OF RESULTS}

Matlab software is a fundamental tool that is often used to solve power system problems and at it will be used to solve this one as well. The results are presented in table 2 .

According to the optimization results, all generators tend to give output as close to its minimum or maximum as possible. Total load must be equal to specific demand according to (6). The first Lagrange multiplier $\lambda$ associated with equality constraint increases along with specific demand.

It is interesting to observe the Lagrange multipliers $\beta$ and their relation to the slack variables and generator output levels. First we will examine the values of $\beta_{i}^{\min }$ in relation to the associated values of $y_{i}$, at chosen load demand of $300 \mathrm{MW}$. All generator outputs are above their lower constraints except generators " 4 " and "9". Consequently, all $y_{i}$, except $y_{4}$ and $y_{9}$ are nonzero, and the square of their values is equal to the generator output in order to satisfy (8). However, since generators "4" and "9" are held at theirs lower limit, $y_{4}=y_{9}=0$. The values of $\beta_{i}^{\text {min }}$ associated with values of $y_{i}$ portray the effect of relaxing the lower limit associated with these constraints 
[3]. Since all other generators are above their lower limits, increasing the lower limit from zero will not make any difference to the value of (5), hence, the value of $\beta^{\text {min }}$ associated with all generators but " 4 " and "9" is zero.

\begin{tabular}{|c|c|c|c|c|c|c|}
\hline \multirow[b]{2}{*}{$i$} & \multicolumn{6}{|c|}{ Load demand for quadratic cost functions } \\
\hline & $180[\mathrm{MW}]$ & $\begin{array}{c}220 \\
{[\mathrm{MW}]}\end{array}$ & $300[\mathrm{MW}]$ & $350[\mathrm{MW}]$ & $450[\mathrm{MW}]$ & $550[\mathrm{MW}]$ \\
\hline $\mathbf{P}_{\mathrm{G} 1}$ & 12.00 & 12.00 & 12.00 & 12.00 & 12.00 & 12.00 \\
\hline $\mathbf{P}_{\mathrm{G} 2}$ & 10.05 & 14.85 & 20.00 & 20.00 & 20.00 & 20.00 \\
\hline $\mathbf{P}_{\mathrm{G} 3}$ & 28.00 & 28.00 & 28.00 & 28.00 & 28.00 & 28.00 \\
\hline $\mathbf{P}_{\mathrm{G} 4}$ & 10.00 & 10.00 & $\underline{\underline{10.00}}$ & 19.67 & 54.19 & 55.00 \\
\hline $\mathbf{P}_{\mathrm{G} 5}$ & 24.38 & 46.59 & 56.00 & 56.00 & 56.00 & 56.00 \\
\hline $\mathbf{P}_{\mathrm{G} 6}$ & 30.55 & 43.55 & 64.70 & 76.00 & 76.00 & 76.00 \\
\hline $\mathbf{P}_{\mathrm{G} 7}$ & 20.00 & 20.00 & 47.55 & 66.28 & 84.00 & 84.00 \\
\hline $\mathbf{P}_{\mathbf{G 8}}$ & 25.00 & 25.00 & 41.74 & 52.04 & 99.81 & 100.00 \\
\hline $\mathbf{P}_{\mathrm{G} 9}$ & 20.00 & 20.00 & 20.00 & 20.00 & 20.00 & 119.00 \\
\hline total & 180 & 220 & 300 & 350 & 450 & 550 \\
\hline $\mathbf{y}_{1}$ & 3.10 & 3.10 & 3.10 & 3.10 & 3.10 & 3.10 \\
\hline $\mathbf{y}_{2}$ & 2.46 & 3.29 & 4.00 & 4.00 & 4.00 & 4.00 \\
\hline $\mathbf{y}_{3}$ & 4.58 & 4.58 & 4.58 & 4.58 & 4.58 & 4.58 \\
\hline $\mathbf{y}_{4}$ & 0.00 & 0.00 & $\underline{0.00}$ & -3.11 & 6.65 & 6.71 \\
\hline $\mathbf{y}_{5}$ & 3.22 & 5.71 & 6.48 & 6.48 & 6.48 & 6.48 \\
\hline$y_{6}$ & 3.92 & 5.32 & 7.04 & 7.80 & 7.80 & 7.80 \\
\hline $\mathbf{y}_{7}$ & 0.00 & 0.00 & 5.25 & 6.80 & 8.00 & 8.00 \\
\hline $\mathbf{y}_{8}$ & 0.00 & 0.00 & 4.09 & 5.20 & 8.65 & 8.66 \\
\hline $\mathbf{y}_{9}$ & 0.00 & 0.00 & $\underline{0.00}$ & 0.00 & 0.00 & 9.95 \\
\hline $\mathbf{x}_{1}$ & 0.00 & 0.00 & 0.00 & 0.00 & 0.00 & 0.00 \\
\hline $\mathbf{x}_{2}$ & 3.15 & 2.27 & 0.00 & 0.00 & 0.00 & 0.00 \\
\hline $\mathbf{x}_{3}$ & 0.00 & 0.00 & 0.00 & 0.00 & 0.00 & 0.00 \\
\hline $\mathbf{x}_{4}$ & 6.71 & 6.71 & 6.71 & 5.94 & 0.90 & 0.00 \\
\hline $\mathbf{x}_{5}$ & 5.62 & 3.07 & 0.00 & 0.00 & 0.00 & 0.00 \\
\hline$x_{6}$ & 6.74 & 5.70 & 3.36 & 0.00 & 0.00 & 0.00 \\
\hline $\mathbf{x}_{7}$ & 8.00 & 8.00 & 6.04 & 4.21 & 0.00 & 0.00 \\
\hline $\mathbf{x}_{8}$ & 8.66 & 8.66 & 7.63 & 6.92 & 0.43 & 0.00 \\
\hline$x_{9}$ & 10.49 & 10.49 & 10.49 & 10.49 & 10.49 & 3.32 \\
\hline$\lambda$ & 496.57 & 678.18 & 980.10 & 1171.6 & 2076.6 & 2673.4 \\
\hline$\beta_{1}{ }^{\min }$ & 0.00 & 0.00 & $\underline{0.00}$ & 0.00 & 0.00 & 0.00 \\
\hline $\boldsymbol{\beta}_{2}{ }^{\min }$ & 0.00 & 0.00 & $\underline{0.00}$ & 0.00 & 0.00 & 0.00 \\
\hline $\boldsymbol{\beta}_{3}{ }^{\min }$ & 0.00 & 0.00 & $\underline{0.00}$ & 0.00 & 0.00 & 0.00 \\
\hline $\boldsymbol{\beta}_{4}{ }^{\min }$ & 423.04 & 241.43 & -60.48 & 0.00 & 0.00 & 0.00 \\
\hline$\beta_{5}{ }^{\min }$ & 0.00 & 0.00 & $\underline{0.00}$ & 0.00 & 0.00 & 0.00 \\
\hline$\beta_{6}{ }^{\min }$ & 0.00 & 0.00 & $\underline{0.00}$ & 0.00 & 0.00 & 0.00 \\
\hline$\beta_{7}{ }^{\min }$ & 204.23 & 22.62 & $\underline{0.00}$ & 0.00 & 0.00 & 0.00 \\
\hline$\beta_{8}{ }^{\min }$ & 175.22 & -6.39 & 0.00 & 0.00 & 0.00 & 0.00 \\
\hline$\beta_{9}{ }^{\min }$ & 514.27 & 332.66 & 30.75 & -160.7 & -1065.8 & 0.00 \\
\hline$\beta_{1}{ }^{\max }$ & 161.94 & 343.55 & 645.46 & 836.95 & 1742.0 & 2338.8 \\
\hline $\boldsymbol{\beta}_{2}{ }^{\max }$ & 0.00 & 0.00 & 106.52 & 298.01 & 1203.0 & 1799.8 \\
\hline$\beta_{3}{ }^{\max }$ & 224.65 & 406.26 & 708.18 & 899.66 & 1804.7 & 2401.5 \\
\hline$\beta_{4}{ }^{\max }$ & 0.00 & 0.00 & 0.00 & 0.00 & 0.00 & 575.29 \\
\hline$\beta_{5}{ }^{\max }$ & 0.00 & 0.00 & 224.26 & 415.74 & 1320.78 & 1917.5 \\
\hline$\beta_{6}{ }^{\max }$ & 0.00 & 0.00 & 0.00 & 26.98 & 932.02 & 1528.8 \\
\hline$\beta_{7}{ }^{\max }$ & 0.00 & 0.00 & 0.00 & 0.00 & 722.51 & 1319.3 \\
\hline$\beta_{8}{ }^{\max }$ & 0.00 & 0.00 & 0.00 & 0.00 & 0.00 & 593.18 \\
\hline$\beta_{9}{ }^{\max }$ & 0.00 & 0.00 & 0.00 & 0.00 & 0.00 & 0.00 \\
\hline
\end{tabular}

Tab. 2. Power generation of $\mathrm{P}_{\mathrm{Gi}}$ for quadratic cost function of a generator

Power generation of each generator in correlation to specific load demand is shown in Fig. 1. Generators "1", " 3 ", "4" and " 5 " operate at their upper limit and all other generator operate below their upper limits. Therefore $x_{1}$ $=x_{3}=x_{4}=x_{5}=0$ in order to satisfy (9). The values of associated $\beta_{i}^{\max }(i=1,3,4,5)$ indicates the sensitivity of (5) to the relaxation of related generator output $P_{G i}$ [3]. For other generators which are operating below their upper limits, the values of $x$ are equal to the square root of the difference between the upper limit and the generator output. Consequently, the values of $\beta_{i}^{\max }$ for these generators are zero. The defined cost functions are quadratic. If we are unable to precisely determine the coefficient $c$ in our cost functions, we can simplify the cost function by letting the coefficient $c_{i}$ tend to zero and therefore making the function linear.

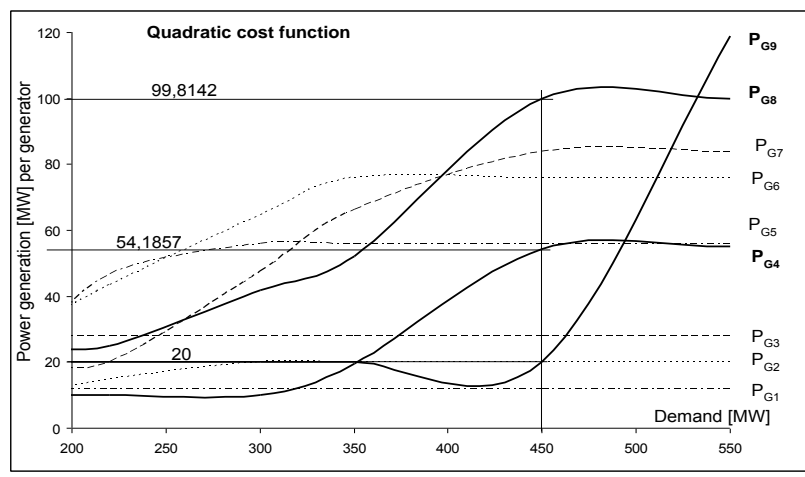

Fig 1. Power generation of each unit in correlation to specific demand for quadratic cost function

With these simplified cost functions we carry out the minimization procedure. The computed results of $P_{\text {Gis }}$ and the differences between quadratic cost function and simplified model are presented in table 3 . This time Lagrange multipliers $\lambda, \beta_{i}^{\min }$ and $\beta_{i}^{\max }$ and variables $\mathrm{y}_{\mathrm{i}}$ and $\mathrm{x}_{\mathrm{i}}$ are not incorporated.

The generators no longer tend to give output as close to its minimum or maximum. For example, the generator " 8 ", at specific demand of $450 \mathrm{MW}$, with quadratic cost curve has output very close to maximum (99,8142 MW), while at simplified method, its output value is 78,65 MW. For generator "4", same thing happens: at specific demand of $450 \mathrm{MW}$, with quadratic cost curve has output very close to maximum $(54,186 \mathrm{MW})$, while at simplified method, its output value is 37,56 MW. Since total demand of 450 MW must be obtained, the difference in output takes on generator "9": with quadratic cost curve its output is minimal (20 MW), while at simplified method, its output value is 57,79 MW.

\begin{tabular}{|c|c|c|c|c|c|c|}
\cline { 2 - 7 } \multicolumn{1}{c|}{} & \multicolumn{6}{c|}{ Load demand for simplified cost functions } \\
\hline $\boldsymbol{i}$ & $\begin{array}{c}\mathbf{1 8 0} \\
{[\mathbf{M W}]}\end{array}$ & $\begin{array}{c}\mathbf{2 2 0} \\
{[\mathbf{M W}]}\end{array}$ & $\begin{array}{c}\mathbf{3 0 0} \\
{[\mathbf{M W}]}\end{array}$ & $\begin{array}{c}\mathbf{3 5 0} \\
{[\mathbf{M W}]}\end{array}$ & $\begin{array}{c}\mathbf{4 5 0} \\
{[\mathbf{M W}]}\end{array}$ & $\begin{array}{c}\mathbf{5 5 0} \\
{[\mathbf{M W}]}\end{array}$ \\
\hline $\mathbf{P}_{\mathbf{G} \mathbf{1}}$ & 12 & 12 & 12 & 12 & 12 & 12 \\
\hline $\mathbf{P}_{\mathbf{G} 2}$ & 9.990 & 14.690 & 20 & 20 & 20 & 20 \\
\hline $\mathbf{P}_{\mathbf{G} 3}$ & 28 & 28 & 28 & 28 & 28 & 28 \\
\hline $\mathbf{P}_{\mathbf{G} 4}$ & 10 & 10 & 10 & 10 & $\mathbf{3 7 . 5 6 0}$ & 55 \\
\hline $\mathbf{P}_{\mathbf{G} 5}$ & 24.11 & 46.172 & 56 & 56 & 56 & 56 \\
\hline $\mathbf{P}_{\mathbf{G} 6}$ & 30.90 & 44.138 & 66.214 & 76 & 76 & 76 \\
\hline $\mathbf{P}_{\mathbf{G} 7}$ & 20 & 20 & 46.359 & 72.211 & 84 & 84 \\
\hline $\mathbf{P}_{\mathbf{G} 8}$ & 25 & 25 & 41.427 & 55.789 & $\mathbf{7 8 . 6 4 8}$ & 100 \\
\hline $\mathbf{P}_{\mathbf{G} \mathbf{9}}$ & 20 & 20 & 20 & 20 & $\mathbf{5 7 . 7 9 2}$ & 119 \\
\hline total & 180 & 220 & 300 & 350 & 450 & 550 \\
\hline
\end{tabular}

Tab. 3. The computed results of $\mathrm{P}_{\mathrm{Gis}}$

When demand for power generation is low, the differences are non-significant, regardless the generator's output. As we are approaching higher level of power demand, the differences in generator's output increases, the distribution of power generation is changing.

In Fig. 3. the differences for generator 6, 7 and 8 are presented. The solid lines represent curves with quadratic cost function while broken lines represent curves of the simplified model. 


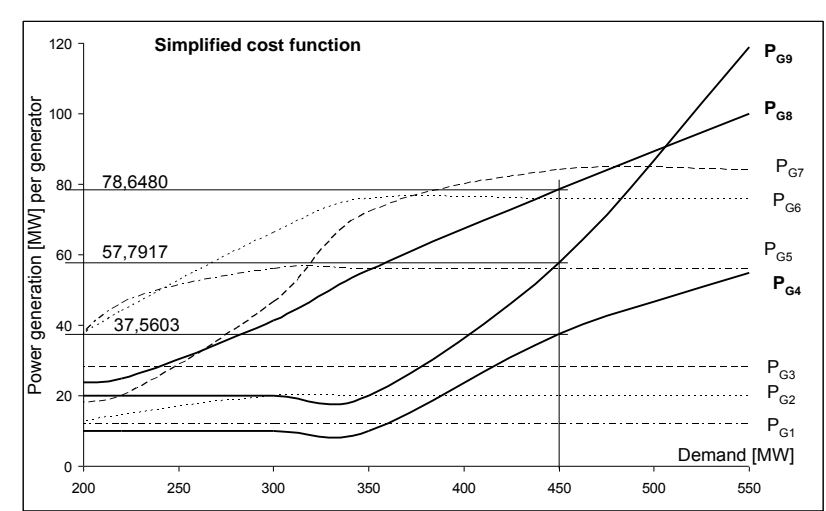

Fig 2. Power generation of each unit in correlation to specific demand for simplified cost function

\begin{tabular}{|c|c|c|c|c|c|c|}
\cline { 2 - 7 } \multicolumn{1}{c|}{} & \multicolumn{7}{c|}{ Related differences $\mathbf{P}_{\mathbf{G i}} \mathbf{-} \mathbf{P}_{\mathbf{G i s}}$} \\
\hline $\boldsymbol{i}$ & $\begin{array}{c}\mathbf{1 8 0} \\
{[\mathbf{M W}]}\end{array}$ & $\begin{array}{c}\mathbf{2 2 0} \\
{[\mathbf{M W}]}\end{array}$ & $\begin{array}{c}\mathbf{3 0 0} \\
{[\mathbf{M W}]}\end{array}$ & $\begin{array}{c}\mathbf{3 5 0} \\
{[\mathbf{M W}]}\end{array}$ & $\begin{array}{c}\mathbf{4 5 0} \\
{[\mathbf{M W}]}\end{array}$ & $\begin{array}{c}\mathbf{5 5 0} \\
{[\mathbf{M W}]}\end{array}$ \\
\hline $\mathbf{1}$ & 0 & 0 & 0 & 0 & 0 & 0 \\
\hline $\mathbf{2}$ & 0.0654 & 0.164 & 0 & 0 & 0 & 0 \\
\hline $\mathbf{3}$ & 0 & 0 & 0 & 0 & 0 & 0 \\
\hline $\mathbf{4}$ & 0 & 0 & 0 & 9.675 & $\mathbf{1 6 . 6 2 5}$ & 0 \\
\hline $\mathbf{5}$ & 0.273 & 0.422 & 0 & 0 & 0 & 0 \\
\hline $\mathbf{6}$ & -0.338 & -0.586 & -1.513 & 0 & 0 & 0 \\
\hline $\mathbf{7}$ & 0 & 0 & 1.199 & -5.931 & 0 & 0 \\
\hline $\mathbf{8}$ & 0 & 0 & 0.314 & -3.744 & $\mathbf{2 1 . 1 6 6}$ & 0 \\
\hline $\mathbf{9}$ & 0 & 0 & 0 & 0 & $\mathbf{- 3 7 . 7 9 2}$ & 0 \\
\hline
\end{tabular}

Tab. 4. Related differences between generator outputs

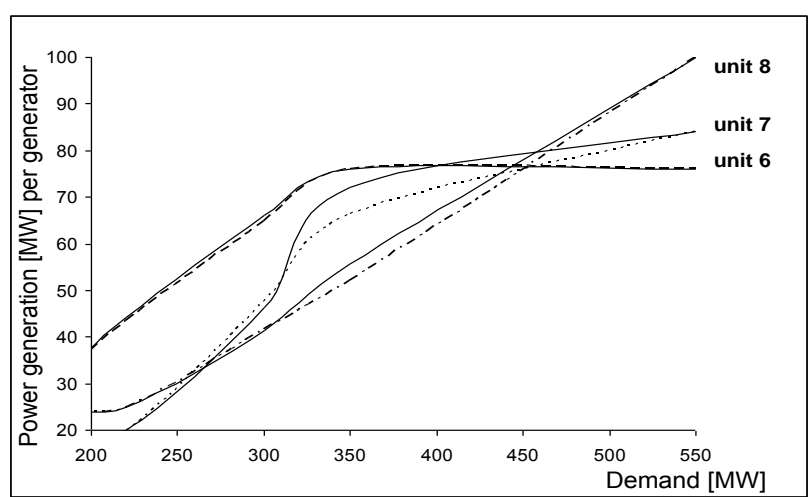

Fig 3. The difference between quadratic and simplified cost functions

\section{CONCLUSION}

The paper describes a method for analyzing the economic dispatch of a power system using Lagrange multiplier method regarding Kuhn-Tucker conditions on quadratic cost functions and simplified model, where quadratic function becomes linear, without taking into consideration the transmission limitations and losses, in order to supply a specific load demand. The optimal solution in generators output obtained from simplified model is not the same as the optimal solution obtained from the exact quadratic fuel cost function. At lower values of specific load demand the differences are not significant, but at higher demand values the power generation distribution is considerably different, especially at larger units. It can be concluded that for higher load demand values we must use as accurate polynomial equations as possible, but for lower load demand values we can use simplified, linear model.

Due to rapid load growth and continuous depletion of fossil fuel reserve, most are looking for non-conventional energy sources as an alternative. One of the solutions is generating power locally at distribution voltage level and thus creating an active distribution network with the possibility of combining multiple and diverse power sources, especially renewable intermittent energy resources like solar, wind and small hydro with nonintermittent ones (gas micro turbines, diesel generators, fuel-cells, biomass thermal power plants etc.).

In presented analysis it is shown that generators tend to operate as close to its constraints, regardless the load demand value. By combining different power sources we are able to use their intermittent characteristic to our advantage. Generators with non-intermittent power sources will mainly operate at maximum output when there is no wind, Sun or water and we can use simplified method. In situation when power generation from intermittent power sources are at maximum output, the generator with non-intermittent power sources will likely be at its minimum or close to its lower limits, which allows as again, to use simplified method.If we use presented simplified linear model we can more easily find and compare the costs of next unit of energy from a generators at given output levels, because incremental cost for all power sources is then a constant. Defining optimum solutions for combined use of power generators regarding working conditions, facility construction, ownership costs and energy efficiency both electrical and heat, are elements for further research.

\section{REFERENCES}

[1] Handschin, E.; Neise, F.; Neumann, H. \& Schultz, R. (2006). Optimal operation of dispersed generation under uncertainty using mathematical programming, International Journal of Electrical Power and Energy Systems, Vol. 28, No. 9, (2006), 618-626, ISSN 0142-0615

[2] Dobren F. A.; Dumitrescu C.D.; Lazarescu C.; Istrat N. \& Amariel, O.I. (2008). Optimization of the production structures, Proceedings of the $19^{\text {th }}$ International DAAAM Symposium, 22-25 $5^{\text {th }}$ October, Trnava, Slovakia, ISSN 1726-9679, ISBN 978-3901509-68-1, Katalinic, B. (Ed.), pp. 0393-0394, Published by DAAAM International, Vienna, Austria

[3] Rau, N. S.(2003). Optimization principles: Practical applications to the Operation and Markets of the Electric Power Industry, IEEE press, Wiley-Interscience, ISBN: 0-471-45130-4, USA

[4] Ogilvie, D. (2010). SYSC-505 Power System Economic Dispatch, Available from: syse.pdx.edu/program/portfolios/.../econdisp.doc Accessed: 2012-04-10

[5] Theerthamalai, A. \& Maheswarapu, S. (2010). An effective noniterative " $\lambda$-logic based" algorithm for economic dispatch of generators with cubic fuel cost function; International Journal of Electrical Power and Energy Systems, Vol. 32, No. 5, (2010), 539-542, ISSN 0142-0615 32

[6] Tang, J.; Cartes, D. \& Baldwin, T. (2003). Economic dispatch with Piecewise linear incremental function and line loss, Power Engineering Society General Meeting, Vol 2. 13-17. July 2003, IEEE, USA, ISBN: 0-7803-7989-6

[7] Alkhalil, D. (2009). Fuel consumption optimization of a multimachines microgrid by secant method combined with IPPD table, International Conference on Renewable Energies and Power Quality (ICREPQ'09) Valencia (Spain), 15-17 April, 2009. ISSN 1213-4171

[8] 2009 Zhai, Q.; Guan, X. \& Yang, J. (2009). Fast commitment based on optimal linear approximation to nonlinear fuel cost: Error analysis and applications, International Journal of Electrical Power Systems Research, Vol. 79, No. 11, (2009), 1604-1613, ISSN 0378-7796

[9] Wang, S.J.; Shahidehpour, S.M.; Kirschemn, D.S.; Mokhtari, S. \& Irisarrvi, G.D. (1995). Short-term generation scheduling with transmission and environmental constraints using an augmented Lagrangian relaxation, IEEE Transactions on Power Systems, (1995), Vol. 19, No. 3, 1294 - 1301, ISSN : 0885-8950 